\title{
$f$-Majorization with Applications to Stochastic Comparison of Extreme Order Statistics
}

\author{
Esmaeil Bashkar \\ Department of Statistics, Yazd University, Yazd, 89175-741, Iran \\ e.bashkar@stu.yazd.ac.ir \\ Hamzeh Torabi \\ Department of Statistics, Yazd University, Yazd, 89175-741, Iran \\ htorabi@yazd.ac.ir \\ Ali Dolati \\ Department of Statistics, Yazd University, Yazd, 89175-741, Iran \\ adolati@yazd.ac.ir \\ Félix Belzunce \\ Department of Statistics and Operations Research, University of Murcia, Murcia, Spain \\ belzunce@um.es \\ mReceived पPI\$ SUO2010 \\ Accepted QR XJ XWR2010
}

\begin{abstract}
In this paper, we use a new partial order, called $f$-majorization order. The new order includes as special cases the majorization, the reciprocal majorization and the $p$-larger orders. We provide a comprehensive account of the mathematical properties of the $f$-majorization order and give applications of this order in the context of stochastic comparison for extreme order statistics of independent samples following the Frèchet distribution and scale model. We discuss stochastic comparisons of series systems with independent heterogeneous exponentiated scale components in terms of the usual stochastic order and the hazard rate order. We also derive new result on the usual stochastic order for the largest order statistics of samples having exponentiated scale marginals and Archimedean copula structure.
\end{abstract}

Keywords: Stochastic order; Exponentiated scale model; Frèchet distribution; Majorization; $f$-majorization order; Archimedean copula.

2000 Mathematics Subject Classification: 60E15, 60K10

\section{Introduction}

In the modern life, applications of order statistics can be found in numerous fields, for example in statistical inference, life testing and reliability theory. The first important work devoted to the stochastic comparisons of order statistics arising from heterogeneous exponential random variables is the one by Pledger and Proschan [33]. Some other papers in this direction, and in particular devoted to the comparison of extreme order statistics from heterogeneous exponential distributions are [12], [34], [19]. There are many other papers on the comparison of extreme order statistics for some other models of parametric distributions. For example [21], [36], [25] and [38] deal with the case of heterogeneous Weibull distributions, [16] and [23] deal with with the case of heterogeneous 
exponentiated Weibull distributions, [3] deals with the case of heterogeneous generalized exponential distributions and [17] deals with the case of heterogeneous Frèchet distributions. A recent review on the topic can be also found in [4].

In these applications, various notions of majorization are used very often. The majorization orders which are used for finding some nice and applicable inequalities is also useful in understanding the insight of the theory. This concept deals with the diversity of the components of a vector in $\mathbb{R}^{n}$. Another interesting weaker order related to the majorization orders introduced in [9] is the $p$-larger order. In [40] the reciprocal majorization is introduced. Note that, for basic notation and terminologies on majorization where we use in this paper, we shall follow [28]. Fang and Zhang [15] and Fang [13] used a notion of majorization to prove Slepian's inequality. In this paper, we use this notion, (called $f$-majorization order) and give applications of this order in the context of stochastic comparison of parallel/series systems with independent and dependent components. This notion includes as particular cases some of the previous ones.

The paper is organized as follows. In Section 2 we provide several notions of stochastic orders and majorization orders, and some known results. We also review notion of $f$-majorization, and then we present the the relationships with the previous notions and some new lemmas that will be used later. In Section 3 we provide new results for the comparison of extreme order statistics from heterogeneous Frèchet, scale and the exponentiated scale populations. We also derive new result on the usual stochastic order for the largest order statistics of the random samples having exponentiated scale marginals and Archimedean copula structure. To finish some conclusions are provided in Section 4.

Throughout this paper, we use the notations $\mathbb{R}=(-\infty,+\infty), \mathbb{R}_{+}=[0,+\infty)$ and $\mathbb{R}_{++}=(0,+\infty)$ and the term increasing means nondecreasing and decreasing means nonincreasing. Also the notation $X_{1: n}\left(X_{n: n}\right)$ is used to denote the smallest (largest) order statistic of $n$ random variables $X_{1}, \ldots, X_{n}$. For any differentiable function $f(\cdot)$, we write $f^{\prime}(\cdot)$ to denote the first derivative. The random variables considered in this paper are all nonnegative.

\section{Preliminaries on majorization and new definitions}

In this section, we recall some notions of stochastic orders, majorization and related orders and some useful lemmas, which are helpful for proving our main results.

Let $X$ and $Y$ be univariate random variables with distribution functions $F$ and $G$, density functions $f$ and $g$, survival functions $\bar{F}=1-F$ and $\bar{G}=1-G$, hazard rate functions $r_{F}=f / \bar{F}$ and $r_{G}=g / \bar{G}$, and reversed hazard rate functions $\tilde{r}_{F}=f / F$ and $\tilde{r}_{g}=g / G$, respectively. Based on these functions several notions of stocahstic orders have been defined, to compare the magnitudes of two random variables. Next we recall some defintions of stocahstic orders that will be used along the paper. For a comprehensive discussion on various stochastic orders, please refer to [30], [35], [26] and more recently [8].

Definition 2.1. Let $X$ and $Y$ be two random variables with common support $\mathbb{R}_{++}$. The random variable $X$ is said to be smaller than $Y$ in the

(i) dispersive order, denoted by $X \leq_{\text {disp }} Y$, if $F^{-1}(\beta)-F^{-1}(\alpha) \leq G^{-1}(\beta)-G^{-1}(\alpha)$ for all $0<\alpha \leq \beta<1$,

(ii) hazard rate order, denoted by $X \leq_{\mathrm{hr}} Y$, if $r_{F}(x) \geq r_{G}(x)$ for all $x$,

(iii) reverse hazard rate order, denoted by $X \leq_{\mathrm{rh}} Y$, if $\tilde{r}_{F}(x) \leq \tilde{r}_{G}(x)$ for all $x$,

(iv) usual stochastic order, denoted by $X \leq_{\text {st }} Y$, if $\bar{F}(x) \leq \bar{G}(x)$ for all $x$. 
A real function $\phi$ is $n$-monotone on $(a, b) \subseteq \mathbb{R}$ if $(-1)^{n-2} \phi^{(n-2)}$ is decreasing and convex in $(a, b)$ and $(-1)^{k} \phi^{(k)}(x) \geq 0$ for all $x \in(a, b), k=0,1, \ldots, n-2$, in which $\phi^{(i)}($.$) is the i$ th derivative of $\phi($.$) . For a n$-monotone $(n \geq 2)$ function $\phi: \mathbb{R}_{+} \longrightarrow[0,1]$ with $\phi(0)=1$ and $\lim _{x \rightarrow+\infty} \phi(x)=0$, let $\psi=\phi,{ }^{-1}$ be the pseudo-inverse, then

$$
C_{\phi}\left(u_{1}, \ldots, u_{n}\right)=\phi\left(\psi\left(u_{1}\right)+\ldots+\psi\left(u_{n}\right)\right), \quad \forall u_{i} \in[0,1], \quad i=1, \ldots, n,
$$

is called an Archimedean copula with the generator $\phi$. Archimedean copulas cover a wide range of dependence structures including the independence copula with the generator $\phi(t)=e^{-t}$. For more on Archimedean copulas, readers may refer to [32] and [29].

Next we provide formal definitions of the different majorization notions that can be found in the literature. Note that, for basic notation and terminologies on majorization used in this paper, we shall follow [28]. To provide these notions, let us recall that the notation $x_{(1)} \leq x_{(2)} \leq \ldots \leq x_{(n)}$ $\left(x_{[1]} \geq x_{[2]} \geq \ldots \geq x_{[n]}\right)$ is used to denote the increasing (decreasing) arrangement of the components of the vector $\boldsymbol{x}=\left(x_{1}, \ldots, x_{n}\right)$.

Definition 2.2. The vector $\boldsymbol{x}$ is said to be

(i) weakly submajorized by the vector $\boldsymbol{y}$ (denoted by $\boldsymbol{x} \preceq_{\mathrm{w}} \boldsymbol{y}$ ) if $\sum_{i=j}^{n} x_{(i)} \leq \sum_{i=j}^{n} y_{(i)}$ for all $j=1, \ldots, n$,

(ii) weakly supermajorized by the vector $\boldsymbol{y}$ (denoted by $\boldsymbol{x} \stackrel{\mathrm{w}}{\preceq} \boldsymbol{y}$ ) if $\sum_{i=1}^{j} x_{(i)} \geq \sum_{i=1}^{j} y_{(i)}$ for all $j=1, \ldots, n$,

(iii) majorized by the vector $\boldsymbol{y}$ (denoted by $\boldsymbol{x} \preceq \boldsymbol{m}$ ) if $\sum_{i=1}^{n} x_{i}=\sum_{i=1}^{n} y_{i}$ and $\sum_{i=1}^{j} x_{(i)} \geq \sum_{i=1}^{j} y_{(i)}$ for all $j=1, \ldots, n-1$.

(iv) A vector $\boldsymbol{x}$ in $\mathbb{R}_{+}^{n}$ is said to be weakly log-majorized by another vector $\boldsymbol{y}$ in $\mathbb{R}_{+}^{n}$ (denoted by $\boldsymbol{x} \preceq_{\mathrm{log}} \boldsymbol{y}$ ) if

$$
\prod_{i=1}^{j} x_{[i]} \leq \prod_{i=1}^{j} y_{[i]}, \quad \forall j=1, \ldots, n
$$

$\boldsymbol{x}$ is said to be $\log$-majorized by $\boldsymbol{y}$ (denoted by $\underset{\log }{\prec} \boldsymbol{y}$ ) if (2.1) holds with equality for $j=n$.

(v) A vector $\boldsymbol{x}$ in $\mathbb{R}_{+}^{n}$ is said to be $p$-larger than another vector $\boldsymbol{y}$ in $\mathbb{R}_{+}^{n}$ (denoted by $\boldsymbol{x} \succeq \boldsymbol{p}$ ) if

$$
\prod_{i=1}^{j} x_{(i)} \leq \prod_{i=1}^{j} y_{(i)}, \quad \forall j=1, \ldots, n
$$

(vi) A vector $\boldsymbol{x}$ in $\mathbb{R}_{+}^{n}$ is said to reciprocal majorized by another vector $\boldsymbol{y}$ in $\mathbb{R}_{+}^{n}$ (denoted by $\boldsymbol{x} \succeq \boldsymbol{r m}$ ) if

$$
\sum_{i=1}^{j} \frac{1}{x_{(i)}} \geq \sum_{i=1}^{j} \frac{1}{y_{(i)}}, \quad \forall j=1, \ldots, n .
$$

The ordering introduced in definition 2.2 (iv), called log-majorization, was defined by Weyl [39] and studied by Ando and Hiai [1], who delved deeply into applications in matrix theory. Note that weak log-majorization implies weak submajorization. See 5.A.2.b. of [28]. Bon and Păltănea 
[9] and Zhao and Balakrishnan [40] introduced the order of $p$-larger and reciprocal majorization, Respectively. Here it should be noted that, for two vectors $\boldsymbol{x}$ and $\boldsymbol{y}$, we have

$$
\boldsymbol{x} \stackrel{\mathrm{p}}{\preceq} \boldsymbol{y} \Longleftrightarrow\left(\log \left(x_{1}\right), \ldots, \log \left(x_{n}\right)\right) \stackrel{\mathrm{w}}{\preceq}\left(\log \left(y_{1}\right), \ldots, \log \left(y_{n}\right)\right) .
$$

It is well-known that (cf. [20] and [22])

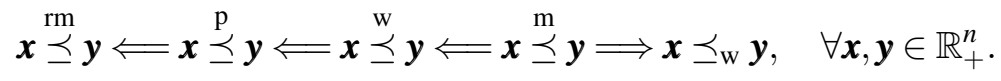

The following lemma is needed for proving the main result.

Lemma 2.1 (Balakrishnan et al. [3]). Let the function $h:(0, \infty) \times(0,1) \longrightarrow(0, \infty)$ be defined as

$$
h(\alpha, t)=\frac{\alpha(1-t) t^{\alpha-1}}{1-t^{\alpha}} .
$$

Then,

(i) for each $0<\alpha \leq 1, h(\alpha, t)$ is decreasing with respect to $t$;

(ii) for each $\alpha \geq 1, h(\alpha, t)$ is increasing with respect to $t$.

We now introduce the main tool for this work. The idea is to use the new majorization notion, used by Fang and Zhang [15] and Fang [13], that includes as particular cases some of the previous ones. Also it will be used to provide some new results for the comparison of extreme values for the Frèchet distribution, scale, and the exponentiated scale model.

Definition 2.3. Let $f: \mathbb{A} \longrightarrow \mathbb{R}$ be a real valued function. The vector $\boldsymbol{x}$ is said to be

(i) weakly $f$-submajorized by the vector $\boldsymbol{y}$, denoted by $\boldsymbol{x} \preceq_{\text {wf }} \boldsymbol{y}$, if $f(\boldsymbol{x}) \preceq_{\mathrm{w}} f(\boldsymbol{y})$

(ii) weakly $f$-supermajorized by the vector $\boldsymbol{y}$, denoted by $\boldsymbol{x} \stackrel{\text { wf }}{\preceq} \boldsymbol{y}$, if $f(\boldsymbol{x}) \stackrel{\mathrm{w}}{\preceq} f(\boldsymbol{y})$

(iii) $f$-majorized by the vector $\boldsymbol{y}$, denoted by $\boldsymbol{x} \preceq \boldsymbol{f m}$, if $f(\boldsymbol{x}) \stackrel{\mathrm{m}}{\preceq} f(\boldsymbol{y})$,

where $f(\boldsymbol{x})=\left(f\left(x_{1}\right), \ldots, f\left(x_{n}\right)\right)$.

It is easy to see that most of the previous majorization notions are examples of the previous notion for some particular choices of the function $f$. In particular we have:

$$
\begin{gathered}
\boldsymbol{x} \preceq \boldsymbol{m} \Longleftrightarrow \boldsymbol{x} \preceq \boldsymbol{f m} \text { when } f(t)=t, \\
\boldsymbol{x} \preceq \boldsymbol{y} \Longleftrightarrow \boldsymbol{x} \preceq \boldsymbol{y} \quad \text { when } f(t)=\log (t), \\
\boldsymbol{x} \preceq \boldsymbol{y} \Longleftrightarrow \boldsymbol{x} \preceq_{\text {wf }} \boldsymbol{y} \quad \text { when } \quad f(t)=\frac{1}{t}, \\
\boldsymbol{x} \preceq_{\log } \boldsymbol{y} \Longleftrightarrow \boldsymbol{x} \preceq_{\text {wf }} \boldsymbol{y} \quad \text { when } \quad f(t)=\log (t) .
\end{gathered}
$$

The following lemma show the relation between $f$-majorization notion and usual majorization for various functions.

\section{Lemma 2.2.}

(i) If an increasing function $f$ is convex, then $\boldsymbol{x} \preceq \boldsymbol{w f}$ implies $\boldsymbol{x} \preceq \boldsymbol{w}$,

(ii) If an increasing function $f$ concave, then $\boldsymbol{x} \preceq_{w f} \boldsymbol{y}$ implies $\boldsymbol{x} \preceq_{w} \boldsymbol{y}$. 
Proof. The proof of this lemma follows easily from Theorem 5.A.2 of [28].

According to Lemma 2.2, all of the results which obtain for weak majorization are also true for $f$-majorization.

An interesting special case of Definition 2.3 by taking the exponential function can be achieved. More precisely, we have the following definition.

Definition 2.4. A vector $\boldsymbol{x}$ in $\mathbb{R}_{+}^{n}$ is said to be weakly exp-majorized by another vector $\boldsymbol{y}$ in $\mathbb{R}_{+}^{n}$ (denoted by $\boldsymbol{x} \underset{\exp }{\mathrm{w}} \boldsymbol{y}$ ) if

$$
\sum_{i=j}^{n} e^{x_{(i)}} \leq \sum_{i=j}^{n} e^{y_{(i)}}, \quad \forall j=1, \ldots, n
$$

$\boldsymbol{x}$ is said to be exp-majorized by $\boldsymbol{y}$ (denoted by $\underset{\exp }{\preceq} \boldsymbol{y}$ ) if (2.2) holds with equality for $j=1$.

Note that weak sub-majorization implies weak exp-majorization. See 5.A.2.g. of [28]. In the following example we see that weak exp-majorization does not imply weak sub-majorization.

Example 2.1. Let $\left(x_{1}, x_{2}\right)=(0.5,0.9)$ and $\left(y_{1}, y_{2}\right)=(1.08,0.3)$. Obviously $\left(x_{1}, x_{2}\right) \succeq_{\mathrm{w}}\left(y_{1}, y_{2}\right)$ and $\left(y_{1}, y_{2}\right) \succeq_{\mathrm{w}}\left(x_{1}, x_{2}\right)$, even though we have $\left(x_{1}, x_{2}\right) \underset{\exp }{\swarrow_{\mathrm{w}}}\left(y_{1}, y_{2}\right)$.

Next we provide an example, which shows that $\boldsymbol{x} \preceq \boldsymbol{f m}$ does not imply $\boldsymbol{x} \preceq \boldsymbol{m}$.

Example 2.2. Let $\boldsymbol{x}=(\sqrt{2}, 5)$ and $\boldsymbol{y}=(2, \sqrt{23})$, then $\stackrel{y}{\text { fm }} \boldsymbol{x}$ with $f(t)=t^{2}$, but it is clear that $\boldsymbol{y}$ is not majorized by $\boldsymbol{x}$.

The following example shows that $\boldsymbol{x} \preceq$ wf $\boldsymbol{y}$ does not imply $\boldsymbol{x} \stackrel{\mathrm{w}}{\preceq} \boldsymbol{y}$, necessarily.

Example 2.3. Let $\boldsymbol{x}=(2,3)$ and $\boldsymbol{y}=(1,5)$, and $f$ be any increasing function that assigns

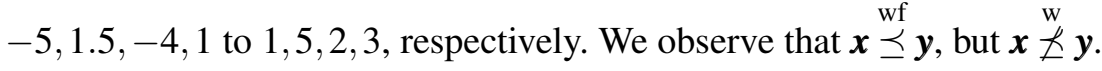

Next we provide a set of technical results that will be used along the paper. First we introduce a lemma, which will be needed to prove our main results and is of interest in its own right.

Lemma 2.3. The function $\varphi: \mathbb{R}_{+}^{n} \longrightarrow \mathbb{R}$ satisfies

(i)

$$
\boldsymbol{x} \preceq_{\mathrm{wf}}(\stackrel{\mathrm{wf}}{\preceq}) \boldsymbol{y} \Longrightarrow \varphi(\boldsymbol{x}) \leq \varphi(\boldsymbol{y})
$$

if and only if, $\varphi\left(f^{-1}\left(a_{1}\right), \ldots, f^{-1}\left(a_{n}\right)\right)$ is Schur-convex in $\left(a_{1}, \ldots, a_{n}\right)$ and increasing (decreasing) in $a_{i}$, for $i=1, \ldots, n$,

(ii)

$$
\boldsymbol{x} \preceq \boldsymbol{y} \Longrightarrow \varphi(\boldsymbol{x}) \leq(\geq) \varphi(\boldsymbol{y})
$$

if and only if, $\varphi\left(f^{-1}\left(a_{1}\right), \ldots, f^{-1}\left(a_{n}\right)\right)$ is Schur-convex (Schur-concave) in $\left(a_{1}, \ldots, a_{n}\right)$, where $a_{i}=f\left(x_{i}\right)$, for $i=1, \ldots, n$ and $f^{-1}(y)=\inf \{x \mid f(x) \geq y\}$. 
Proof. (i) Using definition 2.3, we see that (2.3) is equivalent to

$$
\boldsymbol{a} \preceq_{\mathrm{w}}(\stackrel{\mathrm{W}}{\complement}) \boldsymbol{b} \Longrightarrow \varphi\left(f^{-1}\left(a_{1}\right), \ldots, f^{-1}\left(a_{n}\right)\right) \leq \varphi\left(f^{-1}\left(b_{1}\right), \ldots, f^{-1}\left(b_{n}\right)\right),
$$

where $a_{i}=f\left(x_{i}\right)$ and $b_{i}=f\left(y_{i}\right)$, for $i=1, \ldots, n$. Taking

$$
\phi\left(a_{1}, \ldots, a_{n}\right)=\varphi\left(f^{-1}\left(a_{1}\right), \ldots, f^{-1}\left(a_{n}\right)\right),
$$

in Theorem 3.A.8 of [28], we get the required result.

(ii) This case can be proved in a very similar manner.

It is noteworthy that Lemma 2.1 provided by Khaledi and Kochar [20] is a special case of Lemma 2.3 (i) when $f(x)=\log (x)$ and is useful for proving stochastic orders, see [18] and [3]. Recall that a real valued function $\varphi$ defined on a set $\mathscr{A} \in \mathbb{R}^{n}$ is said to be Schur-convex (Schurconcave) on $\mathscr{A}$ if

$$
\boldsymbol{x} \preceq \boldsymbol{y} \quad \text { on } \quad \mathscr{A} \Longrightarrow \varphi(x) \leq(\geq) \varphi(\boldsymbol{y}) .
$$

\section{Applications to the comparison of extreme order statistics}

In this section we provide new results for the comparison of extreme values from independent Frèchet distribution, scale and exponentiated scale model. We also derive new result on the usual stochastic order for largest order statistics of samples having exponentiated scale marginals and Archimedean copula structure. As we will see the main tools are the new $f$-majorization notions introduced in the previous section.

\subsection{Comparison of extreme order statistics for the Frèchet distribution}

A random variable $X$ is said to be distributed according to the Frèchet distribution, and will be denoted by $X \sim \operatorname{Frè~}(\mu, \lambda, \alpha)$, if the distribution function is given by

$$
G(x ; \mu, \lambda, \alpha)=\exp \left\{-\left(\frac{x-\mu}{\lambda}\right)^{-\alpha}\right\}, \quad x>\mu,
$$

where $\mu \in \mathbb{R}$ is a location parameter, $\lambda>0$ is a scale parameter and $\alpha>0$ is a shape parameter.

In this section, we discuss stochastic comparisons of series and parallel systems with Frèchet distributed components in terms of the hazard rate order and the reverse hazard rate order. The result established here strengthens and generalizes some of the results of [17]. To begin with we present a generalization of Theorem 2 of [17] where sufficient condition is based on the weak $f$ majorization. This theorem provides the stochastic comparison result for the lifetime of the parallel systems having independently distributed Frèchet components with varying scale parameters, but fixed location and shape parameters.

Theorem 3.1. Let $X_{1}, \ldots, X_{n}\left(X_{1}^{*}, \ldots, X_{n}^{*}\right)$ be independent random variables where $X_{i} \sim$ $\operatorname{Frè~}\left(\mu, \lambda_{i}, \alpha\right)\left(X_{i}^{*} \sim \operatorname{Frè~}\left(\mu, \lambda_{i}^{*}, \alpha\right)\right), i=1, \ldots, n$. Let us consider an strictly decreasing (increasing) function $f$.

(i) If $\left(f^{-1}(\cdot)\right)^{\prime}\left(f^{-1}(\cdot)\right)^{\alpha-1}$ is increasing (decreasing) and $\left(\lambda_{1}, \ldots, \lambda_{n}\right) \succeq\left(\lambda_{1}^{*}, \ldots, \lambda_{n}^{*}\right)$ then $X_{n: n} \geq_{\mathrm{rh}}\left(\leq_{\mathrm{rh}}\right) X_{n: n}^{*}$. 
(ii) If $\left(f^{-1}(\cdot)\right)^{\prime}\left(f^{-1}(\cdot)\right)^{\alpha-1}$ is decreasing (increasing) and $\left(\lambda_{1}^{*}, \ldots, \lambda_{n}^{*}\right) \succeq_{\mathrm{wf}}\left(\lambda_{1}, \ldots, \lambda_{n}\right)$ then $X_{n: n} \leq_{\mathrm{rh}}\left(\geq_{\mathrm{rh}}\right) X_{n: n}^{*}$.

Proof. (i) Let us consider a fixed $x>0$, and a strictly monotone function $f$, then the reversed hazard rate of $X_{n: n}$ is given by

$$
\tilde{r}_{X_{n: n}}(x ; \mu, \boldsymbol{a}, \alpha)=\sum_{i=1}^{n} \frac{\alpha}{f^{-1}\left(a_{i}\right)}\left(\frac{x-\mu}{f^{-1}\left(a_{i}\right)}\right)^{-\alpha-1}, \quad x>\mu .
$$

From Lemma 2.3, the proof follows if we prove that, for each $x>0, \tilde{r}_{X_{n: n}}(x ; \mu, \boldsymbol{a}, \alpha)$ is Schur-convex (Schur-concave) and decreasing (increasing) in $a_{i}$ 's.

Let $h\left(a_{i}\right)=\alpha(x-\mu)^{-\alpha-1}\left(f^{-1}\left(a_{i}\right)\right)^{\alpha}$. By the assumption, $f$ is a strictly decreasing (increasing) function, therefore we have

$$
\frac{\partial h\left(a_{i}\right)}{\partial a_{i}}=\alpha^{2}(x-\mu)^{-\alpha-1} \frac{\partial f^{-1}\left(a_{i}\right)}{\partial a_{i}}\left(f^{-1}\left(a_{i}\right)\right)^{\alpha-1} \leq(\geq) 0 .
$$

Hence the reverse hazard rate function of $X_{n: n}$ is decreasing (increasing) in each $a_{i}$.

Now, from Proposition 3.C.1 of [28], the Schur-convexity (Schur-concavity) of $\tilde{r}_{X_{n: n}}(x ; \mu, \boldsymbol{a}, \alpha)$, follows if we prove the convexity (concavity) of $h$. The convexity (concavity) of $h$ follows from the assumption $\left(f^{-1}(\cdot)\right)^{\prime}\left(f^{-1}(\cdot)\right)^{\alpha-1}$ is increasing (decreasing). This completes the proof of the required result.

(ii) The proof is similar to the proof of part (i) and hence is omitted.

Let us describe some particular cases of previous theorem.

In Theorem 3.1, if we let $f(x)=\frac{1}{x}$, we can get the following corollary that generalizes the corresponding result in Theorem 2 of [17]. In particular the majorization assumption is relaxed to the weak majorization and the usual stochastic order is replaced by the stronger reversed hazard rate order.

Corollary 3.1. Let $X_{1}, \ldots, X_{n}\left(X_{1}^{*}, \ldots, X_{n}^{*}\right)$ be independent random variables where $X_{i} \sim$ Frè $\left(\mu, \lambda_{i}, \alpha\right)\left(X_{i}^{*} \sim \operatorname{Frè~}\left(\mu, \lambda_{i}^{*}, \alpha\right)\right), i=1, \ldots, n$. If $\left(\frac{1}{\lambda_{1}}, \ldots, \frac{1}{\lambda_{n}}\right) \succeq \frac{\mathrm{w}}{\succeq}\left(\frac{1}{\lambda_{1}^{*}}, \ldots, \frac{1}{\lambda_{n}^{*}}\right)$ then $X_{n: n} \geq_{\mathrm{rh}} X_{n: n^{*}}^{*}$

In Theorem 3.1, if we let $f(x)=x^{r}, \quad r>0$, we can easily get the following result.

Corollary 3.2. Let $X_{1}, \ldots, X_{n}\left(X_{1}^{*}, \ldots, X_{n}^{*}\right)$ be independent random variables where $X_{i} \sim$ $\operatorname{Frè~}\left(\mu, \lambda_{i}, \alpha\right)\left(X_{i}^{*} \sim \operatorname{Frè~}\left(\mu, \lambda_{i}^{*}, \alpha\right)\right), i=1, \ldots, n$.

(i) If $\alpha \geq r$, and $\left(\lambda_{1}^{r}, \ldots, \lambda_{n}^{r}\right) \succeq_{\mathrm{w}}\left(\left(\lambda_{1}^{*}\right)^{r}, \ldots,\left(\lambda_{n}^{*}\right)^{r}\right)$ then $X_{n: n} \geq_{\mathrm{rh}} X_{n: n}^{*}$.

(ii) If $0<\alpha \leq r$ and $\left(\lambda_{1}^{r}, \ldots, \lambda_{n}^{r}\right) \succeq^{\mathrm{W}}\left(\left(\lambda_{1}^{*}\right)^{r}, \ldots,\left(\lambda_{n}^{*}\right)^{r}\right)$ then $X_{n: n} \leq_{\mathrm{rh}} X_{n: n}^{*}$.

In the next corollary, which is an immediate consequence of Corollary 3.2, we discuss the stochastic comparison of two maximum order statistics, one from a heterogeneous population and the other one from a homogeneous population. Heterogeneity (or homogeneity) of a population is considered with respect to the scale parameters.

Corollary 3.3. Let $X_{1}, \ldots, X_{n}\left(X_{1}^{*}, \ldots, X_{n}^{*}\right)$ be independent random variables where $X_{i} \sim$ Frè $\left(\mu, \lambda_{i}, \alpha\right), i=1, \ldots, n\left(X_{i}^{*} \sim \operatorname{Frè~}(\mu, \lambda, \alpha)\right)$. 
(i) If $\alpha \geq r$, and $\lambda \leq\left(\overline{\lambda^{r}}\right)^{\frac{1}{r}}$ then $X_{n: n} \geq_{\mathrm{rh}} X_{n: n^{*}}^{*}$.

(ii) If $0<\alpha \leq r$ and $\lambda \geq\left(\bar{\lambda}^{r}\right)^{\frac{1}{r}}$ then $X_{n: n} \leq_{\mathrm{rh}} X_{n: n}^{*}$,

where $\overline{\lambda^{r}}=\frac{\sum_{i=1}^{n} \lambda_{i}^{r}}{n}, r>0$.

The first (second) part of the above Corollary gives a lower (upper) bound on the reversed hazard rate function of a parallel system with non-identical components in terms of the one with i.i.d. components when the common scale parameter is $\left(\bar{\lambda}^{-}\right)^{\frac{1}{r}}$. The new bounds are better than the one that follows from the usual majorization which is in terms of the arithmetic mean of the scale parameters since $\tilde{r}_{X_{n: n}}(x ; \mu, \lambda, \alpha)$ is a increasing function of $\lambda$ and the fact that According to the Lyapunov theorem [27], the arithmetic mean of the $\lambda_{i}$ 's is smaller (larger) than $\left(\bar{\lambda}^{-}\right)^{\frac{1}{r}}$ when $r>1(r<1)$.

The following theorem present a generalization of Theorem 1 of [17] where sufficient condition is based on the weak submajorization and by Lemma 2.2 (ii) is true under weak $f$ submajorization for any increasing concave function $f$ of the location parameters.

Theorem 3.2. Let $X_{1}, \ldots, X_{n}\left(X_{1}^{*}, \ldots, X_{n}^{*}\right)$ be independent random variables where $X_{i} \sim$ $\operatorname{Frè~}\left(\mu_{i}, \lambda, \alpha\right)\left(X_{i}^{*} \sim \operatorname{Frè~}\left(\mu_{i}^{*}, \lambda, \alpha\right)\right), i=1, \ldots, n$. If $\left(\mu_{1}, \ldots, \mu_{n}\right) \succeq_{\mathrm{w}}\left(\mu_{1}^{*}, \ldots, \mu_{n}^{*}\right)$, then $X_{n: n} \geq_{\mathrm{rh}} X_{n: n}^{*}$.

Proof. It can be seen that the reversed hazard rate of $X_{n: n}$ is given by

$$
\tilde{r}_{X_{n: n}}(x ; \boldsymbol{\mu}, \lambda, \alpha)=\sum_{i=1}^{n} \frac{\alpha}{\lambda}\left(\frac{x-\mu_{i}}{\lambda}\right)^{-\alpha-1}, \quad x>\max \left(\mu_{1}, \ldots, \mu_{n}\right) .
$$

From Theorem 3.A.8 of [28], the proof follows if we prove that $\tilde{r}_{X_{n: n}}(x ; \boldsymbol{\mu}, \lambda, \alpha)$ is Schur-convex and increasing in $\mu_{i}$ 's.

Let

$$
h\left(\mu_{i}\right)=\frac{\alpha}{\lambda}\left(\frac{x-\mu_{i}}{\lambda}\right)^{-\alpha-1}
$$

then we have

$$
\frac{\partial h\left(\mu_{i}\right)}{\partial \mu_{i}}=\frac{\alpha}{\lambda^{-\alpha}}(\alpha+1)\left(x-\mu_{i}\right)^{-\alpha-2} \geq 0 .
$$

Therefore the reverse hazard rate function of $X_{n: n}$ is increasing in each $\mu_{i}$.

Now, from Proposition 3.C.1 of [28], we only need to prove the convexity of $h$ to get the Schurconvexity of $\tilde{r}_{X_{n: n}}(x ; \boldsymbol{\mu}, \lambda, \alpha)$.

In this case, we have that

$$
\frac{\partial^{2} h\left(\mu_{i}\right)}{\partial \mu_{i}^{2}}=\frac{\alpha}{\lambda^{-\alpha}}(\alpha+1)(\alpha+2)\left(x-\mu_{i}\right)^{-\alpha-3}
$$

Therefore we have that $h$ is a convex function. This completes the proof.

Note that $\left(\mu_{1}, \ldots, \mu_{n}\right) \stackrel{\mathrm{m}}{\succeq}\left(\mu_{1}^{*}, \ldots, \mu_{n}^{*}\right)$ implies $\left(\mu_{1}, \ldots, \mu_{n}\right) \succeq_{\mathrm{w}}\left(\mu_{1}^{*}, \ldots, \mu_{n}^{*}\right)$, Theorem 3.2 substantially improves Theorem 1 of [17]. 


\subsection{Comparison of extreme values for scale model}

Independent random variables $X_{1}, \ldots, X_{n}$ are said to belong to the scale family of distributions if $X_{i} \sim G\left(\lambda_{i} x\right)$ where $\lambda_{i}>0, i=1, \ldots, n$ and $G$ is called the baseline distribution and is an absolutely continuous distribution function with density function $g$. In the Theorem 3.3 we extend result of Theorem 2.1 of [18] to the case when the two sets of scale parameters weakly majorize each other instead of usual majorization which by Lemma 2.2 is true under weak $f$ majorization of the scale parameters.

Theorem 3.3. Suppose $X_{i}$ and $X_{i}^{*}$ as in the setting of Theorem 3.3. If $x r(x)$ is increasing in $x, x^{2} r^{\prime}(x)$ is decreasing (increasing) in $x$ and $\left(\lambda_{1}, \ldots, \lambda_{n}\right) \succeq^{\mathrm{w}}\left(\succeq_{\mathrm{w}}\right)\left(\lambda_{1}^{*}, \ldots, \lambda_{n}^{*}\right)$, then

(i) $X_{1: n} \geq_{\mathrm{hr}}\left(\leq_{\mathrm{hr}}\right) X_{1: n}^{*}$, and

(ii) if $r(x)$ is decreasing then $X_{1: n} \geq_{\operatorname{disp}}\left(\leq_{\mathrm{disp}}\right) X_{1: n}^{*}$.

Proof. (i) Fix $x>0$. Then the hazard rate of $X_{1: n}$ is

$$
r_{X_{1: n}}(x, \boldsymbol{\lambda})=\sum_{i=1}^{n} \lambda_{i} r\left(\lambda_{i} x\right)=\frac{\sum_{i=1}^{n} \varphi\left(\lambda_{i} x\right)}{x},
$$

where $\varphi(u)=u r(u), u \geq 0$. From Theorem 3.A.8 of [28], it suffices to show that, for each $x>0$, $r_{X_{1: n}}(x, \boldsymbol{\lambda})$ is Schur-concave (Schur-convex) and increasing in $\lambda_{i}$ 's. By the assumptions, $\varphi(u)$ is increasing in $u$, then the hazard rate function of $X_{1: n}$ is increasing in each $\lambda_{i}$.

Now, from Proposition 3.C.1 of [28], the concavity (convexity) of $\varphi\left(\lambda_{i} x\right)$ is needed to prove Schur-concavity (Schur-convexity) of $r_{X_{1: n}}(x, \boldsymbol{\lambda})$. Note that the assumption that $u^{2} r^{\prime}(u)$ is decreasing (increasing) in $u$ is equivalent to $r(u)+u r^{\prime}(u)$ is decreasing (increasing) in $u$ since

$$
\left[u^{2} r^{\prime}(u)\right]^{\prime}=u\left(2 r^{\prime}(u)+u r^{\prime \prime}(u)\right)=u\left[r(u)+u r^{\prime}(u)\right]^{\prime},
$$

and $r(u)+u r^{\prime}(u)$ is decreasing (increasing) in $u$ is equivalent to $u r(u)$ is concave (convex) in $u$ since

$$
[u r(u)]^{\prime}=r(u)+u r^{\prime}(u) .
$$

Hence, $\varphi(u)$ is concave (convex). This completes the proof of part (i).

(ii) Using the assumption that $r(x)$ is decreasing in $x$ and part (i), the required result follows from Theorem 2.1 in [2] and Theorem 1 in [5].

Note that the conditions of Theorem 3.3 are satisfied by the generalized gamma distribution as [18] proved that for $X \sim G G(p, q), x r(x)$ is an increasing function of $x$ and $x^{2} r^{\prime}(x)$ is an increasing function of $x$ when $p, q>1$ and is a decreasing function of $x$ when $p, q<1$. Recall that a random variable $X$ has a generalized gamma distribution, denoted by $X \sim G G(p, q)$, when its density function has the following form

$$
g(x)=\frac{p}{\Gamma\left(\frac{q}{p}\right)} x^{q-1} e^{-x^{p}}, \quad x>0,
$$

where $p, q>0$ are the shapes parameters. The conditions of Theorem 3.3 are also satisfied by the Weibull distribution because for $X_{i} \sim W(\alpha, \lambda), x r(x)$ is an increasing function of $x$ and $x^{2} r^{\prime}(x)$ is an increasing function of $x$ when $\alpha \geq 1$ and is a decreasing function of $x$ when $\alpha \leq 1$, so Theorem 3.3 is also a generalization of Theorem 2.3 of [21]. 
Lastly, we get some new results on the lifetimes of parallel systems in terms of the usual stochastic order. It is noteworthy that [18] in Theorem 2.1 proved Theorem 3.4 when $f(x)=\log (x)$ and [19] in Theorem 2.2 proved Theorem 3.4 when the baseline distribution in the scale model is exponential and $f(x)=\log (x)$.

Theorem 3.4. Let $X_{1}, \ldots, X_{n}$ be a set of independent nonnegative random variables with $X_{i} \sim$ $G\left(\lambda_{i} x\right), i=1, \ldots, n$. Let $X_{1}^{*}, \ldots, X_{n}^{*}$ be another set of independent nonnegative random variables with $X_{i}^{*} \sim G\left(\lambda_{i}^{*} x\right), i=1, \ldots, n$. If $\left(f^{-1}\right)^{\prime}(y) \tilde{r}\left(f^{-1}(y)\right)$ is decreasing in $y$, where $f$ is a strictly increasing function, then

$$
\left(\lambda_{1}, \ldots, \lambda_{n}\right) \stackrel{\mathrm{wf}}{\succeq}\left(\lambda_{1}^{*}, \ldots, \lambda_{n}^{*}\right) \Longrightarrow X_{n: n} \geq_{\mathrm{st}} X_{n: n}^{*}
$$

Proof. The survival function of $X_{n: n}$ can be written as

$$
\bar{G}_{X_{n: n}}(t, \boldsymbol{a})=1-\prod_{i=1}^{n} G\left(f^{-1}\left(a_{i}\right) t\right)
$$

where $a_{i}=f\left(\lambda_{i}\right)$, for $i=1, \ldots, n$. Using Lemma 2.3, it is enough to show that the function $\bar{G}_{X_{n: n}}(t, \boldsymbol{a})$ given in (3.2) is Schur-convex and decreasing in $a_{i}$ 's. To prove its Schur-convexity, it follows from Theorem 3.A.4. in [28] that we have to show that for $i \neq j$,

$$
\left(a_{i}-a_{j}\right)\left(\frac{\partial \bar{G}_{X_{n: n}}}{\partial a_{i}}-\frac{\partial \bar{G}_{X_{n: n}}}{\partial a_{j}}\right) \geq 0
$$

that is, for $i \neq j$,

$$
\left(a_{i}-a_{j}\right) \prod_{k=1}^{n} G\left(f^{-1}\left(a_{k}\right) t\right)\left(t\left(f^{-1}\right)^{\prime}\left(a_{j}\right) \frac{g\left(f^{-1}\left(a_{j}\right) t\right)}{G\left(f^{-1}\left(a_{j}\right) t\right)}-t\left(f^{-1}\right)^{\prime}\left(a_{i}\right) \frac{g\left(f^{-1}\left(a_{i}\right) t\right)}{G\left(f^{-1}\left(a_{i}\right) t\right)}\right) \geq 0 .
$$

The assumption $\left(f^{-1}\right)^{\prime}(y) \tilde{r}\left(f^{-1}(y)\right)$ is decreasing in $y$ implies that the function $t\left(f^{-1}\right)^{\prime}\left(a_{i}\right) \tilde{r}\left(f^{-1}\left(a_{j}\right) t\right)$ is decreasing in $a_{i}$, for $i=1, \ldots, n$, from which it follows that (3.3) holds. The partial derivative of $\bar{G}_{X_{n: n}}(t, \boldsymbol{a})$ with respect to $a_{i}$ is negative, which in turn implies that the survival function of $X_{n: n}$ is decreasing in $a_{i}$ for $i=1, \ldots, n$. This completes the proof of the required result.

\subsection{Comparison of extreme values for exponentiated scale model}

Recall that random variable $X$ belongs to the exponentiated scale family of distributions if $X \sim$ $H(x)=[G(\lambda x)]^{\alpha}$, where $\alpha, \lambda>0$ and $G$ is called the baseline distribution and is an absolutely continuous distribution function. We denote this family by $\operatorname{ES}(\alpha, \lambda)$. Bashkar et al. [6] discussed stochastic comparisons of extreme order statistics from independent heterogeneous exponentiated scale samples. In this section, we provide new results for the comparison of smallest order statistics from samples following exponentiated scale model. In the following theorem, we compare series systems with independent heterogeneous ES components when one of the parameters is fixed, and the results are then developed with respect to the other parameter. Again by Lemma 2.2, this result is true under weak $f$-supermajorization where $f$ is a non-negative strictly increasing convex function. 
Theorem 3.5. Let $X_{1}, \ldots, X_{n}\left(X_{1}^{*}, \ldots, X_{n}^{*}\right)$ be independent random variables with $X_{i} \sim \operatorname{ES}\left(\alpha_{i}, \lambda\right)$ $\left(X_{i}^{*} \sim \mathrm{ES}\left(\alpha_{i}^{*}, \lambda\right)\right), i=1, \ldots, n$. Then, for any $\lambda>0$, we have

$$
\left(\alpha_{1}, \ldots, \alpha_{n}\right) \stackrel{\mathrm{w}}{\succeq}\left(\alpha_{1}^{*}, \ldots, \alpha_{n}^{*}\right) \Longrightarrow X_{1: n} \leq_{\mathrm{hr}} X_{1: n}^{*} .
$$

Proof. Fix $x>0$. Then the hazard rate of $X_{1: n}$ is

$$
r_{X_{1: n}}(x, \boldsymbol{\alpha}, \lambda)=\sum_{i=1}^{n} \alpha_{i} \lambda g(\lambda x) \frac{(G(\lambda x))^{\alpha_{i}-1}}{1-(G(\lambda x))^{\alpha_{i}}}=\lambda r(\lambda x) \sum_{i=1}^{n} \varphi\left(\alpha_{i}, G(\lambda x)\right)
$$

where $\varphi(x, p)=\frac{x p^{x}}{1-p^{x}}, x \geq 0,0 \leq p<1$. From Theorem 3.A.8 of [28], it suffices to show that, for each $x>0, r_{X_{1: n}}(x, \boldsymbol{\alpha}, \lambda)$ is Schur-convex and decreasing in $\alpha_{i}$ 's. By the Lemma 2.8 of [37], $\varphi(x, p)$ is decreasing and convex in $x \geq 0$, then the hazard rate function of $X_{1: n}$ is decreasing and convex in each $\alpha_{i}$. So, from Proposition 3.C.1 of [28], the Schur-convexity of $r_{X_{1: n}}(x, \boldsymbol{\alpha}, \lambda)$ follows from convexity of $\varphi(x, p)$. This completes the proof of the Required result.

Recall that, a random variable $X$ is said to be distributed according the generalized exponential distribution, and will be denoted by $X \sim G E(\alpha, \lambda)$, if the distribution function is given by

$$
G(x)=(1-\exp \{-\lambda x\})^{\alpha}, \quad x>0,
$$

where $\alpha>0$ is a shape parameter and $\lambda>0$ is a scale parameter. GE distribution is a member of ES family with underlying distribution $G(x)=1-\exp \{-x\}$. Therefore, we can get the following corollary that generalizes the corresponding result in Theorem 15 of [3]. In particular the majorization assumption is relaxed to the weak supermajorization.

Corollary 3.4. For $i=1, \ldots, n$, let $X_{i}$ and $X_{i}^{*}$ be two sets of mutually independent random variables with $X_{i} \sim \operatorname{GE}\left(\alpha_{i}, \lambda\right)$ and $X_{i}^{*} \sim \operatorname{GE}\left(\alpha_{i}^{*}, \lambda\right)$. Then, for any $\lambda>0$, we have

$$
\left(\alpha_{1}, \ldots, \alpha_{n}\right) \stackrel{\mathrm{w}}{\succeq}\left(\alpha_{1}^{*}, \ldots, \alpha_{n}^{*}\right) \Longrightarrow X_{1: n} \leq_{\mathrm{hr}} X_{1: n}^{*} .
$$

The following result considers the comparison on the lifetimes of series systems in terms of the usual stochastic order when two sets of scale parameters weakly majorize each other.

Theorem 3.6. Let $X_{1}, \ldots, X_{n}\left(X_{1}^{*}, \ldots, X_{n}^{*}\right)$ be independent random variables with $X_{i} \sim \operatorname{ES}\left(\alpha, \lambda_{i}\right)$ $\left(X_{i}^{*} \sim \operatorname{ES}\left(\alpha, \lambda_{i}^{*}\right)\right), i=1, \ldots, n$. If $q(\alpha, x)=\alpha \tilde{r}(x) \frac{G^{\alpha}(x)}{1-G^{\alpha}(x)}$ is decreasing (increasing) in $x$, $\left(\lambda_{1}, \ldots, \lambda_{n}\right) \stackrel{\mathrm{w}}{\succeq}\left(\succeq_{\mathrm{w}}\right)\left(\lambda_{1}^{*}, \ldots, \lambda_{n}^{*}\right)$, then $X_{1: n} \geq_{\mathrm{st}}\left(\leq_{\mathrm{st}}\right) X_{1: n}^{*}$.

Proof. For a fixed $x>0$, the survival function of $X_{1: n}$ can be written as

$$
\left.\bar{F}_{X_{1: n}}(x, \boldsymbol{\lambda})=\prod_{i=1}^{n}\left(1-G\left(\lambda_{i} x\right)\right)^{\alpha}\right) .
$$

Now, using Theorem 3.A.8 of [28], it is enough to show that the function $\bar{F}_{X_{1: n}}(x, \boldsymbol{\lambda})$ given in (3.4) is Schur-convex (Schur-concave) and decreasing in $\lambda_{i}$ 's. 
The partial derivatives of $\bar{F}_{X_{1: n}}(x, \boldsymbol{\lambda})$ with respect to $\lambda_{i}$ is given by

$$
\frac{\partial \bar{F}_{X_{1: n}}(x, \boldsymbol{\lambda})}{\partial \lambda_{i}}=-x \bar{F}_{X_{1: n}}(x, \boldsymbol{\lambda}) q\left(\alpha, \lambda_{i} x\right)
$$

where $q(\alpha, x)=\alpha \tilde{r}(x) \frac{G^{\alpha}(x)}{1-G^{\alpha}(x)}>0$. Then we have that $\bar{F}_{X_{1: n}}(x, \lambda)$ is decreasing in each $\lambda_{i}$.

From Theorem 3.A.4. in [28] the Schur-convexity (Schur-concavity) follows if we prove that, for any $i \neq j$,

$$
\left(\lambda_{i}-\lambda_{j}\right)\left(\frac{\partial \bar{F}_{X_{1: n}}(x, \boldsymbol{\lambda})}{\partial \lambda_{i}}-\frac{\partial \bar{F}_{X_{1: n}}(x, \boldsymbol{\lambda})}{\partial \lambda_{j}}\right) \geq(\leq) 0
$$

that is, for $i \neq j$,

$$
x \bar{F}_{X_{1: n}}(x, \boldsymbol{\lambda})\left(\lambda_{i}-\lambda_{j}\right)\left(q\left(\alpha, \lambda_{j} x\right)-q\left(\alpha, \lambda_{i} x\right)\right) \geq(\leq) 0
$$

By the assumption $q(\alpha, x)$ is decreasing (increasing) in $x$, which in turn implies that the function $q\left(\alpha, \lambda_{i} x\right)$ is decreasing (increasing) in $\lambda_{i}$ for $i=1, \ldots, n$. This completes the proof of the required result.

According to Lemma 2.1, for the GE distribution $q(\alpha, x)=h(\alpha, 1-\exp \{-x\})$ is decreasing (increasing) in $x$ for any $0<\alpha \leq 1(\alpha \geq 1)$, so we have the following corollary.

Corollary 3.5. Let $X_{1}, \ldots, X_{n}\left(X_{1}^{*}, \ldots, X_{n}^{*}\right)$ be independent random variables with $X_{i} \sim G E\left(\alpha, \lambda_{i}\right)$ $\left(X_{i}^{*} \sim G E\left(\alpha, \lambda_{i}^{*}\right)\right), i=1, \ldots, n$. If $0<\alpha \leq 1(\alpha \geq 1)$ and $\left(\lambda_{1}, \ldots, \lambda_{n}\right) \succeq^{\mathrm{w}}\left(\succeq_{\mathrm{w}}\right)\left(\lambda_{1}^{*}, \ldots, \lambda_{n}^{*}\right)$, then $X_{1: n} \geq_{\text {st }}\left(\leq_{\mathrm{st}}\right) X_{1: n}^{*}$

Note that $\left(\lambda_{1}, \ldots, \lambda_{n}\right) \stackrel{\mathrm{m}}{\succeq}\left(\lambda_{1}^{*}, \ldots, \lambda_{n}^{*}\right)$ implies both $\left(\lambda_{1}, \ldots, \lambda_{n}\right) \stackrel{\mathrm{w}}{\succeq}\left(\lambda_{1}^{*}, \ldots, \lambda_{n}^{*}\right)$ and $\left(\lambda_{1}, \ldots, \lambda_{n}\right) \succeq_{\mathrm{w}}\left(\lambda_{1}^{*}, \ldots, \lambda_{n}^{*}\right)$, Corollary 3.5 substantially improves the corresponding ones provided by Balakrishnan et al. [3], in the sense that the majorization is relaxed to the weak majorization. Naturally, one may wonder whether the following two statements are actually also true: (i) For $\alpha \geq 1,\left(\lambda_{1}, \ldots, \lambda_{n}\right) \succeq_{\exp }\left(\lambda_{1}^{*}, \ldots, \lambda_{n}^{*}\right)$ gives rise to the usual stochastic order between $X_{1: n}$ and $X_{1: n}^{*}$; (ii) For $0<\alpha \leq 1,\left(\lambda_{1}, \ldots, \lambda_{n}\right) \stackrel{\mathrm{p}}{\succeq}\left(\lambda_{1}^{*}, \ldots, \lambda_{n}^{*}\right)$ gives rise to the usual stochastic order between $X_{1: n}$ and $X_{1: n}^{*}$. The following example gives negative answers to these two conjectures.

Example 3.1. Let $\left(X_{1}, X_{2}\right)\left(\left(X_{1}^{*}, X_{2}^{*}\right)\right)$ be a vector of independent heterogeneous GE random variables.

(i) Set $\alpha=2,\left(\lambda_{1}, \lambda_{2}\right)=(4,0.5)$ and $\left(\lambda_{1}^{*}, \lambda_{2}^{*}\right)=(2,3)$. Obviously $\left(\lambda_{1}, \lambda_{2}\right) \underset{\exp }{\succeq_{\mathrm{w}}}\left(\lambda_{1}^{*}, \lambda_{2}^{*}\right)$, however $X_{1: 2}$ and $X_{1: 2}^{*}$ are not ordered in the usual stochastic order as can be seen in Fig. 1.

(ii) Set $\alpha=0.6$. For $\left(\lambda_{1}, \lambda_{2}\right)=(1,5.5) \stackrel{\mathrm{p}}{\succeq}(2,3)=\left(\lambda_{1}^{*}, \lambda_{2}^{*}\right), X_{1: 2} \leq_{\mathrm{st}} X_{1: 2}^{*}$; however, for $\left(\lambda_{1}, \lambda_{2}\right)=(1,2.25) \stackrel{\mathrm{p}}{\succeq}(1.1,2.14)=\left(\lambda_{1}^{*}, \lambda_{2}^{*}\right), X_{1: 2} \geq_{\mathrm{st}} X_{1: 2}^{*}$. So, $\left(\lambda_{1}, \lambda_{2}\right) \stackrel{\mathrm{p}}{\succeq}\left(\lambda_{1}^{*}, \lambda_{2}^{*}\right)$ implies neither $X_{1: 2} \leq_{\mathrm{st}} X_{1: 2}^{*}$ nor $X_{1: 2} \geq_{\mathrm{st}} X_{1: 2}^{*}$ for $0<\alpha \leq 1$. 


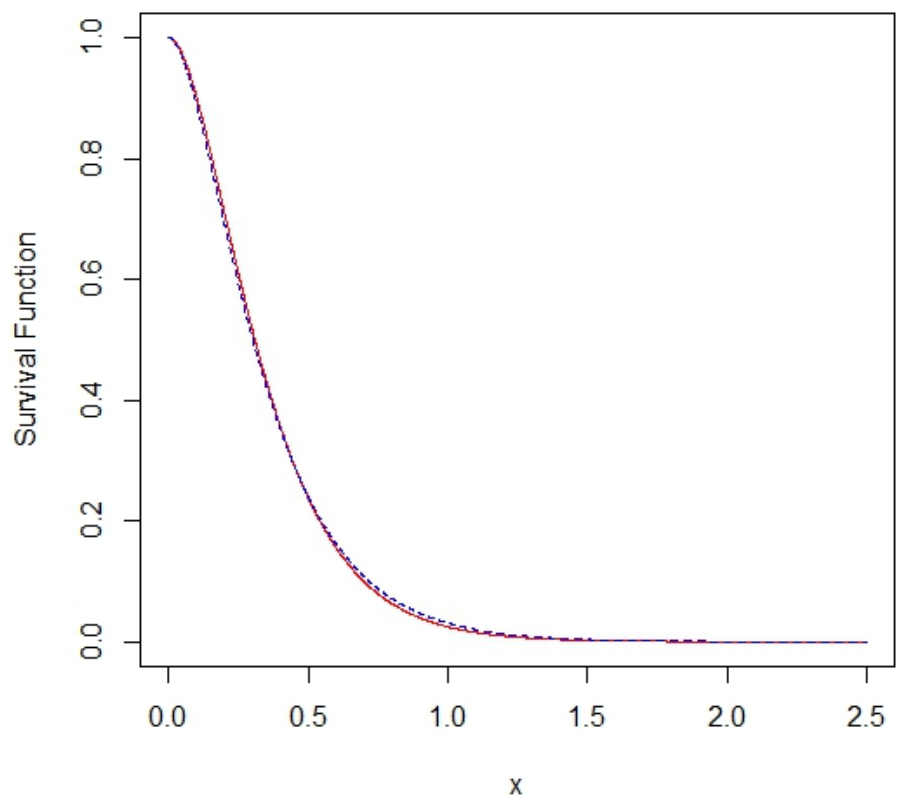

Fig. 1. Plot of the survival functions of $X_{1: 2}$ (dashed line) and $X_{1: 2}^{*}$ (continuous line) when $\alpha=2,\left(\lambda_{1}, \lambda_{2}\right)=(4,0.5)$ and $\left(\lambda_{1}^{*}, \lambda_{2}^{*}\right)=(2,3)$ for random variables with GE distributions.

\subsection{Dependent samples with Archimedean structure}

Recently, some efforts are made to investigate stochastic comparisons on order statistics of random variables with Archimedean copulas. See, for example, [6], [25], [24] and [14]. In this section we derive new result on the usual stochastic order between extreme order statistics of two heterogeneous random vectors with the dependent components having ES marginals and Archimedean copula structure. Specifically, by $\boldsymbol{X} \sim \operatorname{ES}(\boldsymbol{\alpha}, \lambda, \phi)$ we denote the sample having the Archimedean copula with generator $\phi$ and for $i=1, \ldots, n, X_{i} \sim E S\left(\alpha_{i}, \lambda\right)$.

The largest order statistic $X_{n: n}$ of the sample $\boldsymbol{X} \sim \operatorname{ES}\left(\boldsymbol{\alpha}, \lambda, \phi_{1}\right)$ gets distribution function

$$
G_{X_{n: n}}(x)=\phi\left(\sum_{i=1}^{n} \psi\left(G^{\alpha_{i}}(\lambda x)\right)\right)=J(\boldsymbol{\alpha}, \lambda, x, \phi)
$$

Theorem 3.7. For $\boldsymbol{X} \sim \operatorname{ES}\left(\boldsymbol{\alpha}, \lambda, \phi_{1}\right)$ and $\boldsymbol{X}^{*} \sim \operatorname{ES}\left(\boldsymbol{\alpha}^{*}, \lambda, \phi_{2}\right)$,

(i) if $\phi_{1}$ or $\phi_{2}$ is log-convex, and $\psi_{2} \circ \phi_{1}$ is super-additive, then $\left(\alpha_{1}, \ldots, \alpha_{n}\right) \succeq_{\mathrm{w}}\left(\alpha_{1}^{*}, \ldots, \alpha_{n}^{*}\right)$ implies $X_{n: n} \geq_{\mathrm{st}} X_{n: n}^{*}$

(ii) if $\phi_{1}$ or $\phi_{2}$ is log-concave, and $\psi_{1} \circ \phi_{2}$ is super-additive, then $\left(\alpha_{1}, \ldots, \alpha_{n}\right) \stackrel{\mathrm{W}}{\succeq}\left(\alpha_{1}^{*}, \ldots, \alpha_{n}^{*}\right)$ implies $X_{n: n} \leq_{\mathrm{st}} X_{n: n}^{*}$.

Proof. According to Equation (3.6), $X_{n: n}$ and $X_{n: n}^{*}$ have their respective distributin functions $J\left(\boldsymbol{\alpha}, \lambda, x, \phi_{1}\right)$ and $J\left(\boldsymbol{\alpha}^{*}, \lambda, x, \phi_{2}\right)$, for $x \geq 0$. 
(i) We only prove the case that $\phi_{1}$ is log-convex, and the other case can be finished similarly. First we show that $J\left(\boldsymbol{\alpha}, \lambda, x, \phi_{1}\right)$ is decreasing and Schur-concave function of $\alpha_{i}, i=1, \ldots, n$. Since $\phi_{1}$ is decreasing, we have

$$
\begin{gathered}
\frac{\partial J\left(\boldsymbol{\alpha}, \lambda, x, \phi_{1}\right)}{\partial \alpha_{i}}=\log (G(\lambda x))(G(\lambda x))^{\alpha_{i}} \frac{\phi_{1}^{\prime}\left(\sum_{i=1}^{n} \psi_{1}\left(G^{\alpha_{i}}(\lambda x)\right)\right)}{\phi_{1}^{\prime}\left(\psi_{1}\left(G^{\alpha_{i}}(\lambda x)\right)\right)} \leq 0, \\
\text { for all } x>0,
\end{gathered}
$$

That is, $J\left(\boldsymbol{\alpha}, \lambda, x, \phi_{1}\right)$ is decreasing in $\alpha_{i}$ for $i=1, \ldots, n$. Furthermore, for $i \neq j$, the decreasing $\phi_{1}$ implies

$$
\begin{gathered}
\left(\alpha_{i}-\alpha_{j}\right)\left(\frac{\partial J\left(\boldsymbol{\alpha}, \lambda, x, \phi_{1}\right)}{\partial \alpha_{i}}-\frac{\partial J\left(\boldsymbol{\alpha}, \lambda, x, \phi_{1}\right)}{\partial \alpha_{j}}\right)= \\
\left(\alpha_{i}-\alpha_{j}\right) \log (G(\lambda x)) \phi_{1}^{\prime}\left(\sum_{i=1}^{n} \psi_{1}\left(G^{\alpha_{i}}(\lambda x)\right)\right) \\
\left(\frac{(G(\lambda x))^{\alpha_{i}}}{\phi_{1}^{\prime}\left(\psi_{1}\left(G^{\alpha_{i}}(\lambda x)\right)\right)}-\frac{(G(\lambda x))^{\alpha_{j}}}{\phi_{1}^{\prime}\left(\psi_{1}\left(G^{\alpha_{j}}(\lambda x)\right)\right)}\right) \\
\stackrel{\operatorname{sgn}}{=}\left(\alpha_{i}-\alpha_{j}\right)\left(\frac{(G(\lambda x))^{\alpha_{i}}}{\phi_{1}^{\prime}\left(\psi_{1}\left(G^{\alpha_{i}}(\lambda x)\right)\right)}-\frac{(G(\lambda x))^{\alpha_{j}}}{\phi_{1}^{\prime}\left(\psi_{1}\left(G^{\alpha_{j}}(\lambda x)\right)\right)}\right) .
\end{gathered}
$$

where $\stackrel{\text { sgn }}{=}$ means that both sides have the same sign. Note that the log-convexity of $\phi_{1}$ implies the decreasing property of $\frac{\phi_{1}}{\phi_{1}^{\prime}}$. Since $\psi_{1}\left(G^{\alpha_{i}}(x)\right)$ is increasing in $\alpha_{i}$, then $\frac{G^{\alpha_{i}}(x)}{\phi_{1}^{\prime}\left(\psi_{1}\left((G(x))_{i}^{\alpha}\right)\right)}=\frac{\phi_{1}\left(\psi_{1}\left((G(x))^{\alpha_{i}}\right)\right)}{\phi_{1}^{\prime}\left(\psi_{1}\left((G(x))^{\alpha_{i}}\right)\right)}$ is decreasing in $a_{i}$. So, for $i \neq j$,

$$
\left(\alpha_{i}-\alpha_{j}\right)\left(\frac{\partial J\left(\boldsymbol{\alpha}, \lambda, x, \phi_{1}\right)}{\partial \alpha_{i}}-\frac{\partial J\left(\boldsymbol{\alpha}, \lambda, x, \phi_{1}\right)}{\partial \alpha_{j}}\right) \leq 0 .
$$

Then Schur-concavity of $J\left(\boldsymbol{\alpha}, \lambda, x, \phi_{1}\right)$ follows from Theorem 3.A.4. in [28]. According to Theorem 3.A.8 of [28], $\boldsymbol{\alpha} \succeq_{\mathrm{w}} \boldsymbol{\alpha}^{*}$ implies $J\left(\boldsymbol{\alpha}, \lambda, x, \phi_{1}\right) \leq J\left(\boldsymbol{\alpha}^{*}, \lambda, x, \phi_{1}\right)$. On the other hand, since $\psi_{2} \circ \phi_{1}$ is super-additive, by Lemma A.1. of [24], we have $J\left(\boldsymbol{\alpha}^{*}, \lambda, x, \phi_{1}\right) \leq$ $J\left(\boldsymbol{\alpha}^{*}, \lambda, x, \phi_{2}\right)$. So, it holds that

$$
J\left(\boldsymbol{\alpha}, \lambda, x, \phi_{1}\right) \leq J\left(\boldsymbol{\alpha}^{*}, \lambda, x, \phi_{1}\right) \leq J\left(\boldsymbol{\alpha}^{*}, \lambda, x, \phi_{2}\right) .
$$

That is, $X_{n: n} \geq_{\text {st }} X_{n: n}^{*}$.

(ii) We omit its proof due to the similarity to that of Part (i).

Note that Theorem 3.7 for particular case $\lambda=1$ in [14] has been proved.

From Theorem 3.7 (i) and the fact that weak log-majorization implies weak submajorization, we readily obtain the following corollary. 
Corollary 3.6. For $\boldsymbol{X} \sim \operatorname{ES}\left(\boldsymbol{\alpha}, \lambda, \phi_{1}\right)$ and $\boldsymbol{X}^{*} \sim \operatorname{ES}\left(\boldsymbol{\alpha}^{*}, \lambda, \phi_{2}\right)$, if $\phi_{1}$ or $\phi_{2}$ is log-convex, and $\psi_{2} \circ \phi_{1}$ is super-additive, then $\left(\alpha_{1}, \ldots, \alpha_{n}\right) \underset{\log }{\succ_{\mathrm{w}}}\left(\alpha_{1}^{*}, \ldots, \alpha_{n}^{*}\right)$ implies $X_{n: n} \leq_{\text {st }} X_{n: n}^{*}$.

Letting $\lambda=1$ in Corollary 3.6 leads to the following corollary for PRH samples.

Corollary 3.7. For $\boldsymbol{X} \sim \operatorname{PRH}\left(\boldsymbol{\alpha}, \phi_{1}\right)$ and $\boldsymbol{X}^{*} \sim \operatorname{PRH}\left(\boldsymbol{\alpha}^{*}, \lambda, \phi_{2}\right)$,

if $\phi_{1}$ or $\phi_{2}$ is log-convex, and $\psi_{2} \circ \phi_{1}$ is super-additive, then $\left(\alpha_{1}, \ldots, \alpha_{n}\right) \underset{\log }{\succ_{\mathrm{w}}}\left(\alpha_{1}^{*}, \ldots, \alpha_{n}^{*}\right)$ implies $X_{n: n} \leq_{\mathrm{st}} X_{n: n}^{*}$.

Note that [14] in Theorem 5.2 proved the stochastic order between two largest order statistics when $-\log \phi_{1}$ or $-\log \phi_{2}$ is $\log$-concave, but according to Corollary 3.7, we do not need to check the $\log$-concavity of $-\log \phi_{1}$ or $-\log \phi_{2}$ and it is only enough that $\phi_{1}$ or $\phi_{2}$ be $\log$-convex.

\section{Conclusions}

We used a new majorization notion, called $f$-majorization. The new majorization notion includes, as special cases, the usual majorization, the reciprocal majorization and the $p$-larger majorization notions. We provided a comprehensive account of the mathematical properties of the $f$-majorization order and gave applications of this order in the context of stochastic comparison of extreme order statistics.

\section{Acknowledgement}

We would like to express our deep appreciation to referees and Dr Javanshiri for their helpful comments that improved this paper.

\section{References}

[1] T. Ando and F. Hiai, Log majorization and complementary Golden-Thompson type inequalities, Linear Algebra Appl., 197 (1994) 113-131.

[2] I. Bagai and S. C. Kochar, On tail-ordering and comparison of failure rates, Comm. Statist. Theory and Methods, 15(4) (1986) 1377-1388.

[3] N. Balakrishnan, A. Haidari and K. Masoumifard, Stochastic comparisons of series and parallel systems with generalized exponential components, IEEE Trans. Reliab. 64 (2015), 333-348.

[4] N. Balakrishnan and P. Zhao, Ordering properties of order statistics from heterogeneous populations: a review with an emphasis on some recent developments, Probab. Engrg. Inform. Sci. 27 (2013) 403-443.

[5] J. Bartoszewicz, Dispersive ordering and monotone failure rate distributions, Adv. Appl. Probab., (1985) 472-474.

[6] E. Bashkar, H. Torabi and R. Roozegar, Stochastic comparisons of extreme order statistics in the heterogeneous exponentiated scale model, J. Statist. Theory and Appl., 16(2) (2017) 219-238.

[7] G. Barmalzan, A. Haidari and K. Masomifard, Stochastic comparisons of series and parallel systems in the scale model,J. Statist. Sci., 9 (2016) 189-206.

[8] F. Belzunce, C. Martinez-Riquelme and J. Mulero, An Introduction to Stochastic Orders, (ElsevierAcademic Press, London, 2015).

[9] J. L. Bon and E. Păltǎnea, Ordering properties of convolutions of exponential random variables, Lifetime Data Anal. 5 (1999) 185-192.

[10] A. Dolati, On dependence properties of random minima and maxima. Comm. Statist. Theory and Methods, 38 (2008) 393-399. 
[11] A. Dolati, C. Genest and S.C. Kochar, . On the dependence between the extreme order statistics in the proportional hazards model. J. Multivariate Anal., 99 (2008) 777-786.

[12] S. C. Dykstra, S. C. Kochar and J. Rojo, Stochastic comparisons of parallel systems of heterogeneous exponential components, J. Statist. Plann. Inference 65 (1997) 203-211.

[13] L. Fang, Slepian's inequality for Gaussian processes with respect to weak majorization. J. Inequal. Appl., 1 (2013) :5.

[14] R. Fang, C. Li and X. Li, Stochastic comparisons on sample extremes of dependent and heterogenous observations. Statistics, (2015) 1-26.

[15] L. Fang and X. Zhang, Slepian's inequality with respect to majorization. Linear Algebra Appl., 434(4) (2011) 1107-1118.

[16] L. Fang and X. Zhang, Stochastic comparisons of parallel systems with exponentiated weibull components, Statist. Probab. Lett. 97 (2015) 25-31.

[17] N. Gupta, L.K. Patra and S. Kumar, Stochastic comparisons in systems with Frèchet distributed components. Operat. Res. Lett., 43 (2015) 612-615.

[18] B. E. Khaledi, S. Farsinezhad and S. C. Kochar, Stochastic comparisons of order statistics in the scale models, J. Statist. Plann. Inference 141 (2011) 276-286.

[19] B. E. Khaledi and S. C. Kochar, Some new results on stochastic comparisons results, J. Appl. Probab. 37 (2000) 1123-1128.

[20] B. E. Khaledi and S. C. Kochar, Dispersive ordering among linear combinations of uniform random variables, J. Statist. Plann. Inference 100 (2002) 13-21.

[21] B. E. Khaledi and S. C. Kochar, Weibull distribution: some stochastic comparisons results, J. Statist. Plann. Inference 136 (2006) 3121-3129.

[22] S. C. Kochar and M. Xu, On the right spread order of convolutions of heterogeneous exponential random variables, J. Multivariate Anal. 101(1) (2010) 165-179.

[23] A. Kundu and S. Chowdhury, Ordering properties of order statistics from heterogeneous exponentiated weibull models, Statist. Probab. Lett. 114 (2016) 119-127.

[24] X. Li and R. Fang, Ordering properties of order statistics from random variables of Archimedean copulas with applications, J. Multivariate Anal. 133 (2015) 304-320.

[25] C. Li and X. Li, Likelihood ratio order of sample minimum from heterogeneous Weibull random variables. Statist. Probab. Lett., 97 (2015) 46-53.

[26] H. Li and X. Li, Stochastic Orders in Reliability and Risk, (Springer, New York, 2013).

[27] A. M. Lyapunov, Problème général de la stabilité du mouvement. Inn. Fae. Sei. Toulouse Math. 9 (1907) 203-474.

[28] A. W. Marshall, I. Olkin and B. C. Arnold, Inequalities: Theory of Majorization and its Applications, (Springer, New York, 2011).

[29] A. J. McNeil and J. Nešlehová, . Multivariate Archimedean Copulas, d-Monotone Functions and $\ell_{1}$ Norm Symmetric Distributions, Ann. Statist. (2009) 3059-3097.

[30] A. Müller and D. Stoyan, Comparison Methods for Stochastic Models and Risks. (Wiley Series in Probability and Statistics, John Wiley \& Sons, Ltd., Chichester, 2002).

[31] J. Navarro and C. D. Lai, Ordering properties of systems with two dependent components. Comm. Statist. Theory and Methods, 36 (2007) 645-655.

[32] R. B. Nelsen, An Introduction to Copulas (Springer, New York, 2006).

[33] G. Pledger and F. Proschan, Comparisons of order statistics and of spacings from heterogeneous distributions. In: Rustagi, J.S. (Ed.), Optimizing Methods in Statistics. Academic Press, New York, (1971) 89-113.

[34] F. Proschan and J. Sethuraman, Stochastic comparisons of order statistics from heterogeneous populations, with applications in reliability, J. Multivariate Anal. 6 (1976) 608-616.

[35] M. Shaked and J. G. Shanthikumar, Stochastic Orders (Springer, New York, 2007).

[36] L. Fang and W. Tang, On the right spread ordering of series systems with two heterogeneous Weibull components. J. Inequal. Appl., (1) (2014) 190.

[37] N. Torrado, On magnitude orderings between smallest order statistics from heterogeneous beta distributions, J. Math. Anal. Appl. 426(2) (2015) 824-838. 
[38] N. Torrado and S. C. Kochar, Stochastic order relations among parallel systems from Weibull distributions. J. Appl. Probab., 52 (2015) 102-1116.

[39] H. Weyl, Inequalities between the two kinds of eigenvalues of a linear transformation. P. natl. A. Sci., 35 (1949) 408-411.

[40] P. Zhao and N. Balakrishnan, Mean residual life order of convolutions of heterogeneous exponential random variables. J. Multivariate Anal., 100 (2009) 1792-1801. 